\title{
Neurophysiology of Implicit Timing in Serial Choice Reaction-Time Performance
}

\author{
Peter Praamstra, ${ }^{1,2}$ Dimitrios Kourtis, ${ }^{1}$ Hoi Fei Kwok, ${ }^{1}$ and Robert Oostenveld ${ }^{3}$ \\ ${ }^{1}$ Behavioural Brain Sciences Centre, University of Birmingham, Birmingham B15 2TT, United Kingdom, ${ }^{2}$ Department of Neurology, Queen Elizabeth \\ Hospital, Birmingham B15 2TH, United Kingdom, and ${ }^{3}$ F. C. Donders Centre for Cognitive Neuroimaging, 6525 EN Nijmegen, The Netherlands
}

Neural representations of time for the judgment of temporal durations are reflected in electroencephalographic (EEG) slow brain potentials, as established in time production and perception tasks. Here, we investigated whether anticipatory processes in reaction-time procedures are governed by similar mechanisms of interval timing. We used a choice reaction task with two different, temporally regular stimulus presentation regimes, both with occasional deviant interstimulus intervals. Temporal preparation was shown in the form of adjustments in time course of slow brain potentials, such that they reached their maximum amplitude just before a new trial, independent of the duration of the interstimulus interval. Preparation was focused on a brief time window, demonstrated by a drop in amplitude of slow potentials as the standard interval had elapsed in deviant interstimulus intervals. Implicit timing influencing perceptual processing was shown in reduced visual-evoked responses to delayed stimuli after a deviant interstimulus interval and in a reduction of EEG $\alpha$ power over the visual cortex at the time when the standard interval had elapsed. In contrast to explicit timing tasks, the slow brain potential manifestations of implicit timing originated in the lateral instead of the medial premotor cortex. Together, the results show that temporal regularities set up a narrow time window of motor and sensory attention, demonstrating the operation of interval timing in reaction time performance. The divergence in slow brain potential distribution between implicit and explicit timing tasks suggests that interval timing for different behaviors relies on qualitatively similar mechanisms implemented in distinct cortical substrates.

Key words: motor preparation; premotor cortex; electroencephalography; contingent negative variation; reaction time; timing

\section{Introduction}

Timing is essential in the interactions of an organism with its environment, leading to swift adaptation to salient temporal characteristics of the environment. In the laboratory, adjustment to the temporal structure of an experimental task is known to induce temporal preparation that can be measured at the behavioral and the neural levels (Bertelson and Boons, 1960; Trillenberg et al., 2000; Müller-Gethmann et al., 2003; Janssen and Shadlen, 2005; Los and Heslenfeld, 2005). The improvement in performance gained from a predictable, temporally regular task structure is typically attributed to preparation of the motor system (for review, see Müller-Gethmann et al., 2003). Although this association fits a natural propensity for rhythmicity in the motor system (Grosjean et al., 2001), temporal preparation may also be mediated by sensorimotor association (Janssen and Shadlen, 2005) or sensory processes (Ghose and Maunsell, 2002).

Timing functions of the human brain have been investigated almost exclusively with explicit timing tasks. Electroencephalographic (EEG) studies have described a sensitivity to temporal information processing of the contingent negative variation

Received Jan. 31, 2006; revised March 21, 2006; accepted April 12, 2006

We thank Chris Miall and Alan Wing for comments and Nick Roach and Ellen Seiss for technical and programming support.

Correspondence should be addressed to Peter Praamstra, Behavioural Brain Sciences Centre, University of Birmingham, Birmingham B15 2TT, UK. E-mail: p.praamstra@bham.ac.uk.

DOI:10.1523/JNEUROSCI.0440-06.2006

Copyright $\odot 2006$ Society for Neuroscience $\quad$ 0270-6474/06/265448-08\$15.00/0
$(\mathrm{CNV})$, a slow negative wave developing between a warning signal and a subsequent imperative stimulus. A CNV develops when subjects reproduce or judge the duration of a given interval relative to a standard (Walter et al., 1964; Ruchkin et al., 1977; Macar et al., 1999; Pouthas et al., 2000; Pfeuty et al., 2003). Latency and amplitude measures of the CNV correlate with the reproduced duration (Ruchkin et al., 1977) and the judged duration of a stimulus (Macar et al., 1999), respectively. More strikingly, the $\mathrm{CNV}$ that develops during the presentation of a stimulus continues to rise for the duration of a memorized standard, but demonstrates a change in slope when that duration has elapsed and the current stimulus continues (Macar and Vidal, 2003; Pfeuty et al., 2003). Moreover, not only does the CNV peak at the end of a memorized duration, its slope is adjusted such that the CNV peak amplitude at extinction of a memorized standard is the same for short and long standards (Pfeuty et al., 2005). As proposed by Pfeuty et al. (2005), these characteristics of the CNV are in accordance with a neural representation of time intervals by means of "climbing neuronal activity" (Durstewitz, 2003; Reutimann et al., 2004). The medial frontal cortex, in particular the supplementary motor area, has been proposed as the neural substrate of the timing functions reflected in the CNV (Macar et al., 1999; Pfeuty et al., 2005).

The present study investigated whether the implicit timing that is manifested in the automatic adjustment of subjects to the temporal structure of a task is supported by interval timing mechanisms similar to those identified in explicit timing tasks. The 


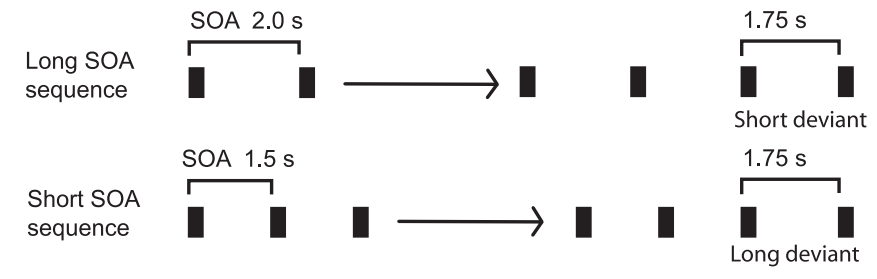

Figure 1. Schematic of the long and short $\mathrm{SOA}$ trial sequences. Stimuli were presented in short series of 11, 13, 15, 17, 19, or 21 trials. Each series of stimuli had SOAs of either 1.5 or $2.0 \mathrm{~s}$, except for the last $\mathrm{SOA}$, which was always $1.75 \mathrm{~s}$.

questions addressed were, first, whether the timing features reflected in slow brain potentials are preserved under implicit timing conditions, and second, whether their distribution supports the same neural substrate as for explicit timing functions. Implicit timing processes were investigated by means of a comparison between two different stimulus presentation regimes and by perturbing a standard interstimulus interval by a deviant interval.

\section{Materials and Methods}

Participants. Ten subjects (seven men, age $34 \pm 9$ years), all of whom were right-handed according to self-report, took part in the experiment. All had normal or corrected-to-normal vision. Informed consent was obtained and the investigation was approved by the department's ethical review board.

Procedures and stimuli. The experiment consisted of a choice response task to arrow stimuli presented on a computer screen. Subjects had to indicate the direction of left or right pointing arrows by pressing one of two buttons. The experiment was divided in eight blocks of $\sim 6$ min each. Within each block, individual trials were presented in series of $11,13,15$, 17,19 , or 21 trials. In each series, the stimulus onset asynchrony (SOA) between successive reaction stimuli was either 1.5 or $2.0 \mathrm{~s}$, except for the last SOA, which was always $1.75 \mathrm{~s}$, which is $250 \mathrm{~ms}$ shorter (short deviant) or longer (long deviant) than the preceding SOAs (Fig. 1). Trial sequences with long and short SOAs followed each other randomly with pauses of $8 \mathrm{~s}$ between them to give subjects time to blink. The total number of sequences presented was 96 , equally divided in short and long SOA series. Responses to each stimulus were made with the index or middle finger depending on the direction of the arrow, which pointed to the left or right with equal probability. Subjects started the experiment with the left or right hand and switched response hand every two blocks. Half of the subjects started with the left, the other half with the right hand. The experiment was preceded by a short practice block that contained sequences with long and short SOAs. Participants were not made aware of the deviant final SOA. They were debriefed after the experiment and asked whether they had been aware of any temporal irregularity other than the different SOAs between series. Subsequently, they were told about the deviant SOAs and asked again whether they had noticed this feature.

The experiment was run in a normally illuminated room. Stimuli were presented on a 17 inch monitor at a resolution of $800 \times 600$. Participants were seated comfortably in an armchair with their eyes $100 \mathrm{~cm}$ from the monitor. Response keys were attached to the armrests of the chair and subjects rested their index and middle fingers on the keys. The stimuli were presented in white on a gray background for a duration of $200 \mathrm{~ms}$. A fixation area was indicated by permanently displayed brackets surrounding the central screen area where the arrow stimuli were presented. The brackets enclosed a square of $1^{\circ} \times 1^{\circ}$ of visual angle; the arrows measured $0.75^{\circ} \times 0.75^{\circ}$ of visual angle.

EEG recording, data processing, and analysis. EEG was recorded continuously with $\mathrm{Ag} / \mathrm{AgCl}$ electrodes from 128 scalp electrodes relative to an (off-line) averaged left and right mastoid reference. The electrodes were placed according to the 10-5 electrode system (Oostenveld and Praamstra, 2001) using a nylon electrode cap. Eye movements were monitored by bipolar horizontal and vertical EOG derivations. EEG and electrooculography (EOG) signals were amplified with a bandpass of $0-128 \mathrm{~Hz}$ by BioSemi Active-Two amplifiers and sampled at $512 \mathrm{~Hz}$. The continuous EEG recordings were off-line segmented in $3000 \mathrm{~ms}$ epochs starting $500 \mathrm{~ms}$ before stimulus onset and spanning one intertrial interval. Subsequently, separate averages were constructed for short and long SOA conditions performed with the left and with the right hand. To capture the preparatory activity at a time when subjects were adjusted to the series' fixed SOA, these averages only included the last eight SOAs (before the final deviant SOA) of each series. Averages of the final deviant SOAs were constructed separately for short deviant and long deviant SOAs, but collapsed across response hands given the small number of deviant trials ( 48 short and 48 long deviants, each divided in 24 trials per hand). For all averages, the prestimulus baseline was aligned to zero, by subtracting the mean signal amplitude in the interval from -500 to $0 \mathrm{~ms}$. For the analysis of event-related potentials, individual trials containing eye movement or blink artifacts were rejected. For analysis of event-related changes in spectral power in Brain Electric Source Analysis (BESA; MEGIS, Gräfelfing, Germany) (see below), eye movement and blink artifact were corrected using a multiple source analysis approach (Berg and Scherg, 1994).

Event-related potential amplitudes were analyzed by pooling the values of neighboring electrodes within regions of interest (ROIs), identified on the basis of the scalp topography. Thus, the amplitude and slope of the $\mathrm{CNV}$ were analyzed in ROIs overlying the left and right premotor cortex (C3/4, FC3/4, FCC3 h/4 h, FCC5 h/6 h). The slope of the CNV was derived from the mean amplitudes measured between 600-700 and 1400-1500 ms (short SOA condition), and from the mean amplitudes measured between 600-700 and 1900-2000 ms (long SOA condition). Analyses on the slope of the $\mathrm{CNV}$ in deviant trials included additional ROIs overlying the centroparietal cortex $(\mathrm{Pz}, \mathrm{POz}, \mathrm{PPO} 1 \mathrm{~h}, \mathrm{PPO} 2 \mathrm{~h})$, and left and right occipitotemporal cortex (PO7/8, P7/8, POO9 h/10 h, PPO9 $\mathrm{h} / 10 \mathrm{~h}$ ). Here, slopes were quantified by fitting a linear regression line to data points between 1600 and $1800 \mathrm{~ms}$.

For the analysis of event-related changes in spectral power, epochs were further processed to obtain temporal spectral evolution (TSE) waveforms (Salmelin and Hari, 1994). To generate TSE waveforms, the individual epochs were first bandpass filtered for frequency bands of interest using Butterworth zero phase-shift filters ( $24 \mathrm{~dB} /$ octave). The filtered epochs were then rectified before averaging to prevent phase cancellation. The resulting averaged waveforms represent the timevarying magnitude of activity in the selected frequency band. This report will refer only to findings in the $\alpha$ band ( $8-12 \mathrm{~Hz}$ ) TSE waveforms that discriminated between the processing of short and long deviant SOAs.

In addition to the TSE analysis, a more comprehensive analysis of event-related spectral perturbations was performed using BESA. After segmentation and artifact correction, each single trial was transformed in the time-frequency domain using complex demodulation set to a frequency resolution of $1 \mathrm{~Hz}$ and temporal resolution of $50 \mathrm{~ms}$ in the frequency range $2-50 \mathrm{~Hz}$. Time-frequency representations per channel and subject were created by averaging spectral density amplitude over trials. Individual subject and grand average representations for short and long SOA conditions revealed a pronounced modulation of $\beta$ activity in the intertrial interval. The $\beta$ activity modulation was first analyzed at the sensor level and then localized using a beamformer analysis. The BESA beamformer is a modified version of the linearly constrained minimum variance vector beamformer in the time-frequency domain as described by Gross et al. (2001). The beamformer operator is computed using the cross-spectral density matrix (the time-frequency equivalent of the data covariance matrix) computed from the single-trial data. This allows one to image evoked as well as induced oscillatory activity in a user-defined time-frequency range, where time is taken relative to a triggered event. In beamforming analyses, the whole brain is scanned voxel by voxel. The activity at each voxel is computed by applying a spatial filter to the data. The time course of $\beta$ modulation was analyzed using an ROI analysis and compared in time windows $850-1150$ and $1150-1450 \mathrm{~ms}$ poststimulus against a $300 \mathrm{~ms}$ prestimulus baseline. Two ROIs were defined in left and right motor cortex, respectively, each including the voxels within a 10 $\mathrm{mm}$ radius of the grand average activation maxima in the contralateral and ipsilateral motor cortex.

The cortical sources of slow brain potentials were estimated with a distributed multiple source modeling technique (minimum norm ap- 
proach), implemented in BESA. The approach involves the computation of the source activities of a large number of regional sources evenly distributed over 1420 standard locations of the smoothed surface of a standard brain. A unique current distribution over this predetermined layer of regional sources, explaining the surface measurements, is obtained by applying the minimum norm constraint, which selects the solution with minimum overall intensity (Hämäläinen and Ilmoniemi, 1994). The obtained solutions were robust under different weighting options.

Reaction-time analyses were performed on the responses to trials after a deviant SOA (short deviant; long deviant), as well as on the eight trials preceding the deviant. The responses to these eight trials were averaged to obtain a mean value for short standards and long standards, respectively. EEG and reaction-time data were analyzed using ANOVA in SigmaScan Pro software (SPSS, Chicago, IL).

\section{Results}

Response times

The participants were debriefed after the experiment to evaluate whether they had been aware of the deviant final SOAs. None of the participants had noticed the timing perturbations. Nonetheless, there was evidence of breaches of temporal expectation reflected in slower reaction times [i.e., $377 \pm 25$ vs $365 \pm 25 \mathrm{~ms}\left(F_{(1,9)}=7.01\right.$; $p<0.05)$ for the "long deviants" relative to their $1.5 \mathrm{~s}$ standards, and $373 \pm 30 \mathrm{vs}$ $365 \pm 25 \mathrm{~ms}\left(F_{(1,9)}=14.90 ; p<0.01\right)$ for the "short deviants" relative to their $2.0 \mathrm{~s}$ standards]. Error rates were between 2.7 and $5.2 \%$ for responses after standard and deviant SOAs, with no significant difference between conditions.

\section{Neurophysiology \\ Effects of SOA on slow preparatory \\ brain potentials}

If CNV-like slow preparatory brain potentials that develop between successive trials are influenced by an implicit timing mechanism similar to what has been described in explicit timing tasks, then their time course should be different for short and long SOA conditions, such that the amplitude reached before the next trial is the same in short and long SOA conditions. In contrast, if they are not under the control of such a timing mechanism, then the amplitude should be higher for long SOAs than for short SOAs, because the CNV will continue to rise. To evaluate these contrasting hypotheses, the $\mathrm{CNV}$ topography was determined in the last $100 \mathrm{~ms}$ preceding the next trial (i.e., in windows from 1400-1500 and 1900-2000 ms for the short and long SOA conditions, respectively). The CNV amplitude was subsequently quantified in two groups of four electrodes over left and right premotor cortex, where the CNV reached its maximum amplitude.

\section{Left hand responding}

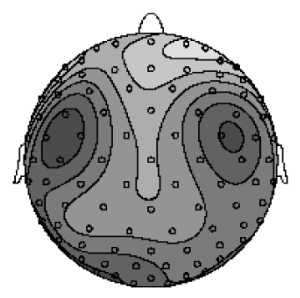

Long SOA

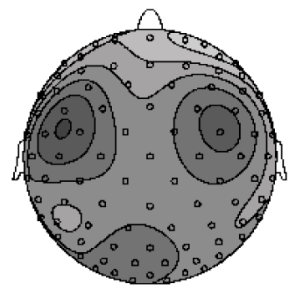

Short SOA

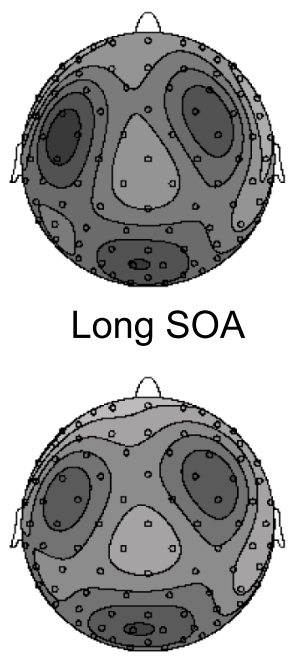

Short SOA

Right hand responding
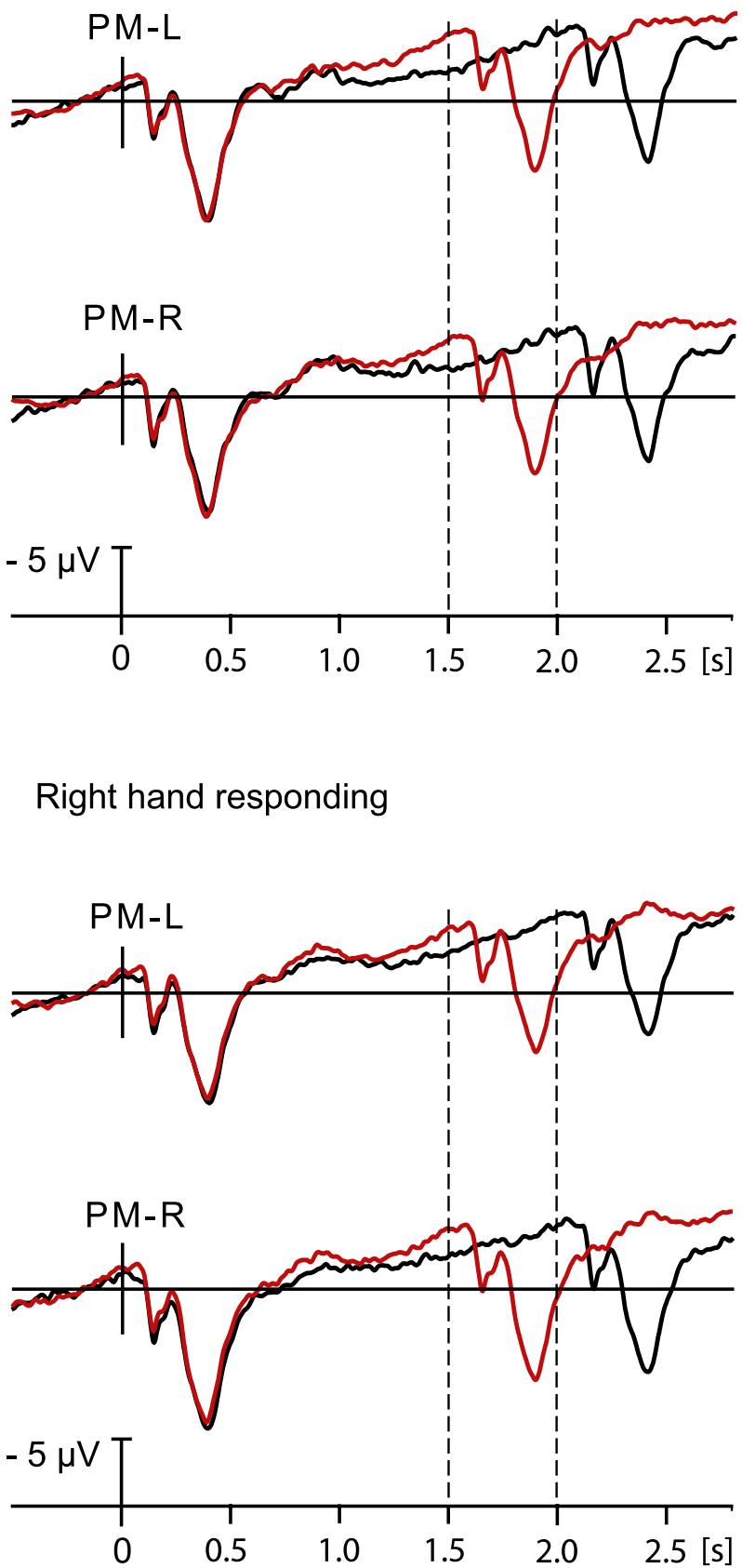

Figure 2. Scalp topography and CNV waveforms in short and long SOA conditions separately for left (top) and right (bottom) response blocks. Waveforms represent averaged activity of four electrodes overlying the left (PM-L) and right (PM-R) premotor cortex activation maxima. Line spacing in the scalp maps is $0.5 \mu \mathrm{V}$. The dashed lines indicate the 1500 and $2000 \mathrm{~ms} \mathrm{SOA} \mathrm{durations.}$

As shown in Figure 2, the CNV time course is adjusted to the duration of the SOA, and the CNV reaches the same amplitude before the next trial regardless of the SOA. This was expressed in an absence of an effect of $\operatorname{SOA}\left(F_{(1,9)}=1.23 ; p=0.30\right)$. Comparing the slope of the short and long SOA conditions (see Materials and Methods), the CNV rose steeper for the short than for the long SOA condition $\left(F_{(1,9)}=7.70 ; p=0.022\right)$. In neither of the analyses did the 


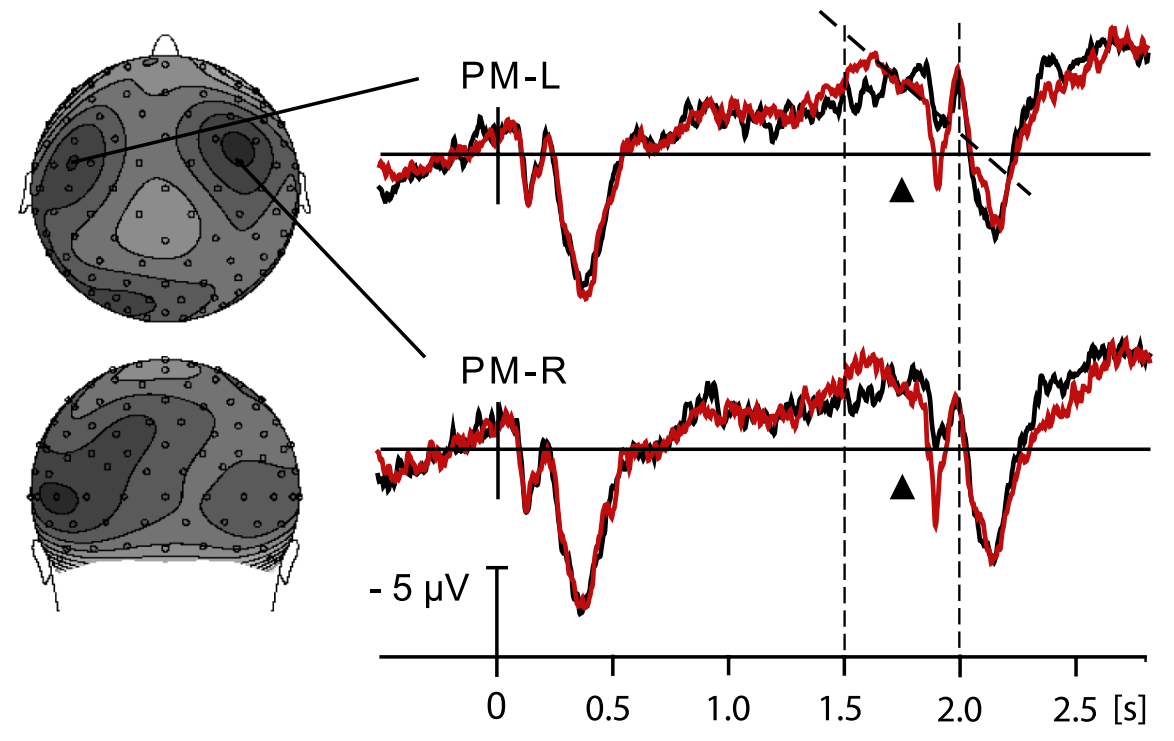

SOA lengthened

Figure 3. Effect of timing perturbation on slow brain potentials. The topography of the CNV in the long deviant condition $(1400-1500 \mathrm{~ms})$ is similar to the topography seen with regular SOA. The CNV over left and right premotor areas peaks at $\sim 1600$ ms (i.e., shortly after the expected time of stimulus arrival) and drops in amplitude before stimulus presentation at $1750 \mathrm{~ms}$ (arrow heads). The dashed lines indicate the expected 1500 and $2000 \mathrm{~ms} \mathrm{SOA} \mathrm{durations.} \mathrm{In} \mathrm{the} \mathrm{top} \mathrm{traces,} \mathrm{a} \mathrm{regression} \mathrm{line} \mathrm{is} \mathrm{fitted} \mathrm{to} \mathrm{the}$ downgoing slope of the CNV in the long deviant condition. Line spacing in the scalp maps is $0.5 \mu \mathrm{V}$.

CNV differ between hemispheres, nor was it modulated by the side of the response $(F$ values $\leq 1)$. The latter finding indicates that the timing reflected in the CNV is not simply preparation of the response hand.

These results show that the CNV developing between successive reaction stimuli reaches a fixed amplitude just before the anticipated termination of the entrained interval. This amplitude is independent of the duration of the interval, because the CNV adjusts its slope to the duration of the interval after repeated exposure. These results resemble findings reported by Pfeuty et al. (2005) in an explicit timing task showing that CNV activity peaks at the end of a memorized duration, and that its slope varies inversely with the length of this duration.

\section{Effects of timing perturbations on slow preparatory brain potentials}

Previous work using explicit timing tasks has found that a test duration that is longer than a memorized standard duration produces a CNV that terminates around the latency where the memorized standard terminates (Macar and Vidal, 2003; Pfeuty et al., 2003). In the present experiment, passive entrainment with a regular interstimulus interval produced a similar modulation of the $\mathrm{CNV}$ in the deviant final interstimulus interval when the deviance consisted of a lengthening of the regular SOA. This is shown in Figure 3, where the CNVs of deviant final SOAs are superimposed. The CNVs of long and short deviant SOAs show a different rise time because of the preceding entrainment with short and long standard SOAs, respectively. The CNV of the short deviant SOA shows a steady rise until the presentation of the reaction stimulus at $1750 \mathrm{~ms}$. Crucially, the CNV of the long deviant SOA reached maximum amplitude around $1600 \mathrm{~ms}$ (i.e., $100 \mathrm{~ms}$ later than the expected arrival of the stimulus, but still 150 ms before the actual stimulus). To quantify the difference between the conditions, the slope of the CNV was measured in the time window from 1600-1800 ms. This analysis was performed on signals from five groups of four electrodes overlying the left and right premotor cortex, the central parietal cortex, and the left and right occipitotemporal cortex, respectively. These five groups of electrodes cover the maxima in the CNV distribution. For the short deviant, a rising slope was found, whereas for the long deviant, there was a down going slope, yielding a main effect of (preceding) SOA $\left(F_{(1,9)}\right.$ $=5.20 ; p<0.05)$. The effect was solely caused by the modulation measured over left and right premotor cortices, as demonstrated by a significant interaction of SOA by area $\left(F_{(1,9)}=9.99 ; p<0.05\right)$ and post hoc tests. For the left and right premotor cortex, post hoc $t$ tests yielded a significant difference in the slope of short and long deviant CNVs $\left(t_{(9)}=3.14, p=0.012\right.$; and $t_{(9)}=3.34, p=0.009$, respectively). For the parietal and occipitotemporal locations, $t$ tests yielded $t$ values $<1.25$, with $p$ values $>0.24$. For the left and right premotor cortex, the difference between the CNVs was also quantified in terms of the CNV peak latency, determined in a search window between 1500 and 1850 ms. The peak latency was found to be significantly earlier for the $\mathrm{CNV}$ in the long deviant condition $\left(F_{(1,9)}=7.35 ; p<0.05\right)$. Together, these findings indicate that temporal deviance is registered in slow brain potential time course over lateral premotor areas. In combination with the results in the previous section, the findings demonstrate the operation of an interval timing process that creates a window of temporal attention with boundaries before and after the expected event.

\section{Effects of SOA on oscillatory activity}

Analyses of slow brain potentials were complemented with analyses of oscillatory EEG activity, which is known to exhibit preparation phenomena in motor (Salmelin et al., 1995; Stancak and Pfurtscheller, 1995) and sensory domains (Worden et al., 2000). Changes in oscillatory activity were evaluated from timefrequency representations, computed between $2-50 \mathrm{~Hz}$ for each channel over the entire duration of short and long SOAs. This analysis revealed a pronounced modulation of $\beta$-band activity over the bilateral sensorimotor cortex, with maximum $\beta$ power in a window of $850-1150 \mathrm{~ms}$ after stimulus for both SOA conditions. Initial analyses of $\beta$ modulation in relevant clusters of electrodes demonstrated differences in time course between the short and long SOA conditions. To capture both time course and localization of the $\beta$ activity modulation, we applied a beamformer analysis to $\beta$-band activity between 14 and $24 \mathrm{~Hz}$ in time windows of $850-1150$ and $1150-1450 \mathrm{~ms}$.

$\beta$ Power peaked in the interval $850-1150 \mathrm{~ms}$ after stimulus, relative to the $\beta$ power before the stimulus. This $\beta$ event-related synchronization (ERS) effect was localized to the bilateral motor cortex centered around Talairach-Tournoux coordinates $x=$ $\pm 30, y=-16$, and $z=56$ (Fig. 4). Analyses across conditions were performed on ROIs centered on the above coordinates, using a four-way ANOVA with factors SOA, epoch, hemisphere, and hand. The analysis showed a main effect of SOA $\left(F_{(1,9)}=\right.$ 27.43; $p<0.001)$ because of a stronger ERS for the long SOA 
condition. Although the $\beta$ power peaked in the early time window, it declined again in the subsequent time window, yielding a main effect of epoch $\left(F_{(1,9)}=7.84 ; p<\right.$ 0.05). Importantly, this decline was steeper for the short SOA condition, resulting in an interaction of SOA by epoch $\left(F_{(1,9)}=21.75 ; p<0.001\right) . \beta$ ERS was strongest over the hemisphere contralateral to the response hand (interaction hand by hemisphere: $F_{(1,9)}=16.49$; $p<$ $0.01)$, especially in the long SOA condition $\left(F_{(1,9)}=24.98 ; p<0.01\right)$.

The increase and subsequent decrease of $\beta$ power represent postmovement $\beta$ synchronization and preparatory desynchronization. The adjustment in the time course of the preparatory desynchronization reflects temporal preparation. The lateralization of this effect to the hemisphere contralateral to movement indicates that the preparation is much closer to the movement execution level than the symmetrical timing effects represented in the CNV.

\section{Effects of timing perturbations on oscillatory activity}

Given the small number of trials with deviant SOA, changes in oscillatory activity could not be analyzed with beamformer source reconstruction. Time-frequency representations were computed between $2-50 \mathrm{~Hz}$ as for the standard SOAs. Eventrelated changes in oscillatory activity were found only in the $\alpha$ band and are here represented by means of TSE waveforms (see Materials and Methods). Because the long and short deviant SOAs were of the same duration (1.75 s), any difference in EEG activity between them can be attributed to the temporal preparation conditioned by the preceding standard SOAs of 1.5 or $2.0 \mathrm{~s}$. The time-varying magnitude of activity in the $\alpha$ band $(8-12 \mathrm{~Hz})$ is illustrated in Figure 5, with TSE traces from the lengthened SOA condition superimposed on those of the shortened SOA condition. The former condition shows a sharp reduction of $\alpha$ activity at the time that subjects expect the arrival of a stimulus (1.5 s). Comparison against the short deviant condition in a statistical parametric map shows that the reduction in $\alpha$ activity is localized over the occipital scalp. The effect was further evaluated from two sets of four pooled electrodes overlying the left and right occiput in a time window of 1.65-1.85 s. An ANOVA with factors hemisphere and deviance demonstrated a significant difference between short and long deviants $\left(F_{(1,9)}=10.22 ; p=0.011\right)$ without hemispheric asymmetry.

Although $\alpha$ EEG activity is suppressed by visual stimulation, suppression in the absence of stimulation must be caused by internal timing, possibly reflecting a temporal attention mechanism. This possibility was supported by an attenuated visualevoked response to the delayed stimulus. This response was smaller than the response to the immediately preceding stimulus
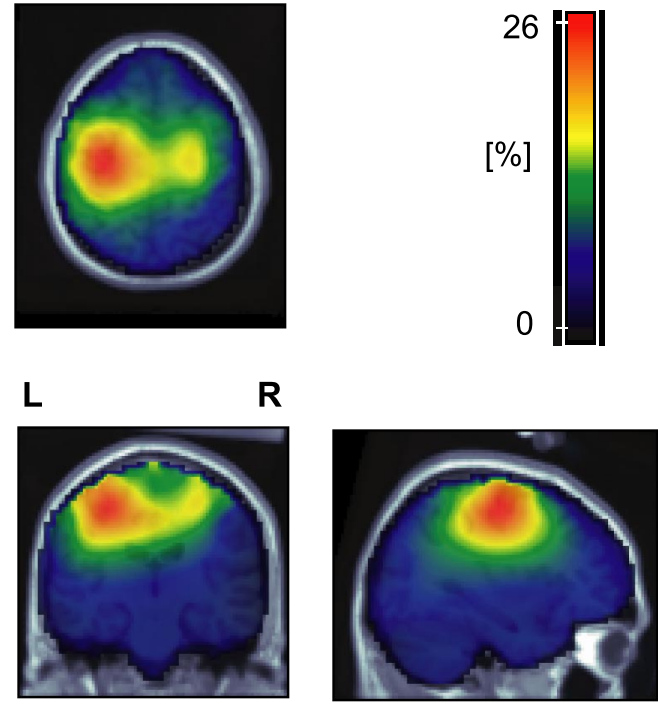

Figure 4. $\quad \beta$-Band $(14-24 \mathrm{~Hz})$ modulation in precentral cortex. The graphs show $\beta$-power change relative to baseline in time windows of $850-1150 \mathrm{~ms}$ (early) and $1150-1450 \mathrm{~ms}$ (late). The grand average beamformer estimate of the $\beta$-power change in the long SOA condition with right-hand response (window 850-1150 ms) is shown. Error bars indicate SEM.

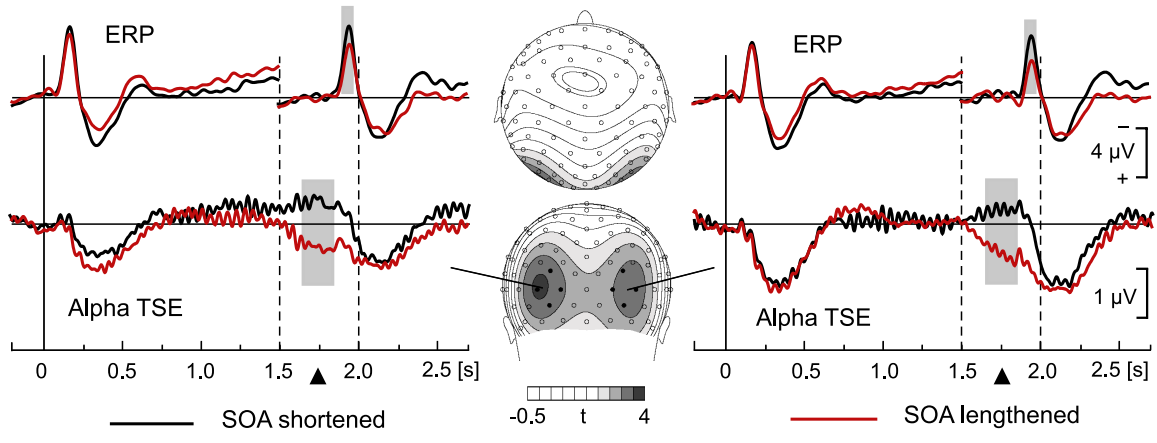

Figure 5. Modulation of visual evoked potentials and $\alpha$ activity. TSE traces represent the temporal evolution of $\alpha$ activity during the deviant final SOAs of $1750 \mathrm{~ms}$ (time indicated by arrowheads). $\alpha$ Activity decreases sharply at $1500 \mathrm{~ms}$ (i.e., the time of the expected stimulus) in the short SOA condition. $\alpha$ Activity continues unchanged when the stimulus is expected at $2000 \mathrm{~ms}$ occipital scalp (uncorrected, two-tailed $t$ test). The four gray shades represent $t$ values between 2.2 and 4.0 (critical $t$ value for $p<$ 0.05: 2.26). The stimulus in the long deviant condition occurring later than anticipated evoked a visual response of reduced amplitude, as illustrated in the ERP traces from four pooled electrodes. For better visualization, the visual-evoked responses are low-pass filtered $(10 \mathrm{~Hz})$ and displayed and quantified relative to a baseline between 1500 and $1750 \mathrm{~ms}$.

at standard $\operatorname{SOA}\left(F_{(1,9)}=8.55 ; p=0.017\right)$ as well as smaller than the response to short deviants $\left(F_{(1,9)}=9.09 ; p=0.015\right)$ (Fig. 5).

\section{Source characterization}

To ascertain the presumed origin of the bilateral frontal maxima of the CNV in the lateral premotor cortex, we performed source analysis using a distributed multiple source modeling approach implemented in BESA. For each individual subject, conditions were pooled by aligning short and long SOA conditions at stimulus onset. These individual datasets were then combined in a grand average across subjects. The computed minimum norm current estimate of distributed activity on the smoothed cortical surface reproduced the bilateral frontal maxima of the scalp distribution. Like the scalp distribution, the minimum norm current estimate was stable over the $500 \mathrm{~ms}$ interval preceding the reaction stimulus (Fig. 6). The centers of the source maxima were located at Talairach-Tournoux coordinates $x=-46, y=-3$, and $z=53$ for the left frontal maximum, and at $x=43, y=-6$, 

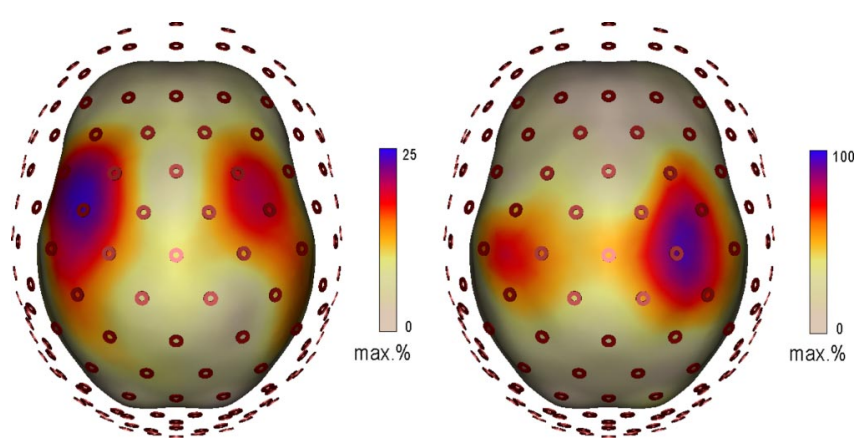

Figure 6. Minimum norm current estimates of the CNV (left) and movement-related activity (right). The current estimate for the CNV refers to a time point $200 \mathrm{~ms}$ before the reaction stimulus. The movement-related sources were estimated at peak latency $40 \mathrm{~ms}$ before the button press. The source locations of the movement-related activity serve as landmarks for verification of the origin of the frontal CNV maxima.

and $z=55$ for the right frontal maximum. These locations are in the anterior zone of the precentral cortex corresponding with premotor cortex.

To lend additional credibility to the above analysis, we used movement-related EEG activity as a landmark for the hand motor cortex. For this purpose, the continuous EEG records were resegmented, time-locked to the subjects' motor responses, and averaged across conditions, separately for left- and right-hand button presses. Finally, the left- and right-hand averages were subtracted to isolate movement-related from overlapping stimulus-related activity. Because of left hemisphere motor dominance and consequent left hemisphere involvement in left-hand movement (Kim et al., 1993), this subtraction shows stronger right than left motor cortex activation (Fig. 6). The activation maxima were at $x=-36, y=-25$, and $z=59$ in the left and at $x=35, y=-22$, and $z=58$ in the right hemisphere. These maxima are in the posterior zone of the precentral cortex corresponding with the primary motor cortex. The accurate localization of movement-related EEG potentials to the hand motor cortex provides support for the frontal CNV maxima originating in the premotor cortex.

The $z$-coordinates of the premotor cortex activation foci correspond with the dorsal rather than the ventral premotor cortex (cf. Schubotz and von Cramon, 2003). Although the discussion below focuses on timing aspects of lateral premotor cortex function revealed by the $\mathrm{CNV}$, it is noteworthy that activation of the dorsal premotor cortex would also be expected on the basis of other features of the task. Both conditional motor tasks and visuospatial information processing invoke the dorsal premotor cortex (Halsband and Passingham, 1985; Toni et al., 1999).

\section{Discussion}

\section{Slow brain potentials and implicit timing}

The results demonstrate that exposition to regular sequences of reaction stimuli conditions temporal expectation, with neurophysiological manifestations that resemble effects found in explicit timing tasks. The CNV developing between successive reaction stimuli reached a fixed amplitude just before the termination of the entrained interval. This amplitude was independent of interval duration because of adjustment of the slope of the CNV. Similar findings were reported by Pfeuty et al. (2005) in a task where subjects compared a test duration against a memorized standard. One could interpret the development of the same amplitude negative shift in short and long SOA conditions as merely reflecting the buildup of a nonspecific state of motor readiness. This interpretation can be rejected on the basis of the slow brain potential time course in long deviant SOAs, where the $\mathrm{CNV}$ reached its peak around the time of the expected stimulus arrival, to drop in amplitude before the delayed stimulus arrived. Again, this behavior is similar to what is observed when a test duration is judged against a memorized standard that is of shorter duration (Macar and Vidal, 2003), demonstrating that the CNV, in these experimental conditions, does not just reflect motor readiness, but expresses the duration of a stored standard interval. As shown here, the standard duration does not need to be learned by explicit memorization of a given time interval, but can consist of a duration acquired through repeated presentation of stimuli and/or repeated actions (i.e., through conditioning).

Whereas the timing properties of slow brain potentials are the same in implicit and explicit timing, their scalp distribution is different. In explicit timing, effects on the CNV have been attributed to the medial premotor cortex or supplementary motor area (SMA) (Macar et al., 1999; Pfeuty et al., 2005). Activation of the SMA in timing tasks is thought to emerge from striatofrontal circuits involved in timing, a hypothesis supported by neuroimaging results (Macar et al., 2002; Ferrandez et al., 2003; Coull et al., 2004). In our implicit timing task, in contrast, the CNV is predominantly caused by activations of the left and right premotor cortex, with an additional focus over the centroparietal scalp. The latter activation likely originates from the left and right superior parietal cortex or from the medial parietal cortex. Activation of the superior parietal cortex is occasionally found in imaging investigations of time perception (Rao et al., 2001) and motor timing (Jantzen et al., 2005). Parietal activation associated with timing is more frequently found in the inferior parietal cortex (Coull et al., 2004), consistent with a sensitivity to elapsed time of the lateral intraparietal cortex in the macaque (Janssen and Shadlen, 2005).

Anticipatory neural activity of the lateral premotor cortex, influenced by event predictability, was described previously in the macaque by Mauritz and Wise (1986). In humans, activation of the lateral premotor cortex in timing tasks has been found most consistently with continuous timing requirements, as in monitoring a rhythm for deviant elements (Schubotz et al., 2000; Schubotz and von Cramon, 2001). This activation is not dependent on motor output and includes both the ventral and dorsal premotor cortex. In these tasks, lateral premotor cortex activation was found accompanied by SMA activation. Lateral premotor cortex activation might serve the coding of interval durations relevant to representational functions of the premotor cortex associated with motor function. For instance, the responsiveness to temporal parameters of sensory events could be invoked for synchronization of motor output to rhythmic events (Schubotz and von Cramon, 2001). Accordingly, the lateral premotor cortex is activated by paced finger tapping (Jäncke et al., 2000). Schubotz and von Cramon (2003) summarized the timing function of the lateral premotor cortex as both extraction and prediction of sequential/temporal information. In this framework, the time course adjustments of the CNV to SOA duration can be regarded as expression of motor prediction. The change in slope of the $\mathrm{CNV}$ in long deviant SOAs, in turn, would reflect the lateral premotor cortex capacity for extracting sequential/temporal information.

\section{Modulation of oscillatory activity and implicit timing}

When comparing oscillatory activity in the standard short and long SOAs, the main finding was an adjustment in time course of $\beta$ activity to the duration of the SOA. Anticipatory decrease of 
oscillatory activity in $\alpha$ and $\beta$ bands has been reported with selfpaced movements (Stancak and Pfurtscheller, 1995) and in stimulus anticipation (Bastiaansen et al., 1999; Worden et al., 2000). $\beta$ Power is also sensitive to the timing of response selection after a task-relevant stimulus (Kaiser et al., 2001). The present results show that the time course of anticipatory $\beta$ activity modulation is adjusted to the timing requirements of a task. $\beta$ Activity sources were identified in the precentral cortex, consistent with previous investigations (Salmelin et al., 1995; van Burik and Pfurtscheller, 1999).

Event-related spectral perturbations in the $\alpha$ band differentiated the short and long deviant conditions. $\alpha$ Activity is normally suppressed by visual stimulation. We attribute the reduction of $\alpha$ activity in the absence of stimulation to temporal attention, as supported by an attenuated visual-evoked response to the delayed stimulus. Hence, implicit timing-based temporal attention acts at least partly on early perceptual processes. Early work on temporal attention failed to find such modulatory effects (Miniussi et al., 1999), but the finding is in agreement with more recent work (Lange et al., 2003; Doherty et al., 2005). Importantly, behavioral effects of subliminal visual stimuli depend on predictable timing or explicit temporal cueing (Naccache et al., 2002), which also indicates that perceptual processing can be amplified by phasic allocation of temporal attention, enabled by regular task timing.

The combined pattern of event-related changes in $\alpha$ band and visual-evoked response amplitude could suggest that the reduction of $\alpha$ activity terminates a state of heightened sensitivity to expected stimulation. However, reduction of $\alpha$ activity is usually considered a prerequisite for active information processing (Fries et al., 2001), and is beneficial to visual detection and discrimination performance (Hanslmayr et al., 2005). It is therefore also possible that reduction of $\alpha$ initiated a facilitation of sensory processing. This is not incompatible with the attenuated visualevoked response elicited while $\alpha$ had not yet returned to baseline. Attentional effects on EEG rhythms include not only amplitude modulation, but also the susceptibility to phase synchronization by a stimulus (Hanslmayr et al., 2005) and even anticipatory phase adjustments (Barry et al., 2004). These more transient effects are critical determinants of evoked-response amplitude (Makeig et al., 2002).

\section{Conclusions}

It has long been recognized that reaction time measures are not only determined by the information and decision making processes inherent in a task, but also by timing mechanisms (MacDonald and Meck, 2004). The present investigation provides neurophysiological evidence for the operation of interval timing mechanisms that are recruited in an implicit manner by the temporally regular structure of a task. Related mechanisms of timebased sensory and motor anticipation may underlie timing in predictive motor control.

The effects of implicit timing on the time course of the CNV replicate similar effects found in explicit timing tasks. However, unlike in explicit timing tasks, these effects originate in lateral premotor structures instead of the medial premotor cortex (Macar et al., 1999; Pfeuty et al., 2005). This divergence suggests that distinct cortical substrates can implement similar timing mechanisms for different behaviors. Possibly, these different cortical substrates show the same effects by virtue of their participation in basal ganglia-thalamocortical circuits that implement a flexible, general-purpose timing function (Buhusi and Meck, 2005). Taking the modulation of occipital $\alpha$-band activity and visual-evoked potentials into account, our data demonstrate anticipatory timing in motor and sensory domains. The data do not establish whether motor and sensory anticipation are coordinated by a central timing mechanism. Hence, it is also possible that implicit timing emerges from distributed neural substrates that do not have a specialized timing function (Mauk and Buonomano, 2004).

As observed previously by Pfeuty et al. (2005), the temporal processing exhibited in the CNV resembles the anticipatory behavior of delay-period sustained activity recorded in neurons from frontal (Niki and Watanabe, 1979; Akkal et al., 2004), parietal (Leon and Shadlen, 2003), and thalamic neurons (Komura et al., 2001). Both demonstrate a temporal profile characterized by climbing neuronal activity (Durstewitz, 2003; Reutimann et al., 2004). Like the CNV, the slope of climbing activity changes as the typical interval between relevant events changes. Moreover, both the CNV and climbing activity suddenly drop when an anticipated stimulus (or predicted reward) does not occur at the expected time (Komura et al., 2001; Durstewitz, 2003). Important with regard to implicit timing, models of climbing activity for the representation of temporal intervals allow for the encoding of time to be incidental rather than intrinsic to a task (Reutimann et al., 2004). This is also an important feature of reward-based learning models using the temporal difference error between the predicted and actual time of reward occurrence (Hollerman and Schultz, 1998; Durstewitz, 2003). In these models, midbrain dopamine neurons fit the role of neurons producing an error signal, thus shaping the climbing profile of anticipatory prediction signals (Suri and Schultz, 2001). Based on such a role of midbrain dopamine neurons, the basal ganglia are in a position to influence implicit timing in a wide range of different behaviors.

\section{References}

Akkal D, Escola L, Bioulac B, Burbaud P (2004) Time predictability modulates presupplementary motor area neuronal activity. NeuroReport 15:1283-1286.

Barry RJ, Rushby JA, Johnstone SJ, Clarke AR, Croft RJ, Lawrence CA (2004) Event-related potentials in the auditory oddball as a function of EEG alpha phase at stimulus onset. Clin Neurophysiol 115:2593-2601.

Bastiaansen MC, Böcker KB, Cluitmans PJ, Brunia CH (1999) Event-related desynchronization related to the anticipation of a stimulus providing knowledge of results. Clin Neurophysiol 110:250-260.

Berg P, Scherg M (1994) A multiple source approach to the correction of eye artifacts. Electroencephalogr Clin Neurophysiol 90:229-241.

Bertelson P, Boons JP (1960) Time uncertainty and choice reaction time. Nature 187:531-532.

Buhusi CV, Meck WH (2005) What makes us tick? Functional and neural mechanisms of interval timing. Nat Rev Neurosci 6:755-765.

Coull JT, Vidal F, Nazarian B, Macar F (2004) Functional anatomy of the attentional modulation of time estimation. Science 303:1506-1508.

Doherty JR, Rao A, Mesulam MM, Nobre AC (2005) Synergistic effect of combined temporal and spatial expectations on visual attention. J Neurosci $25: 8259-8266$.

Durstewitz D (2003) Self-organizing neural integrator predicts interval times through climbing activity. J Neurosci 23:5342-5353.

Ferrandez AM, Hugueville L, Lehericy S, Poline JB, Pouthas V (2003) Basal ganglia and supplementary motor area subtend duration perception: an fMRI study. NeuroImage 19:1532-1544.

Fries P, Reynolds JH, Rorie AE, Desimone R (2001) Modulation of oscillatory neuronal synchronization by selective visual attention. Science 291:1560-1563.

Ghose GM, Maunsell JH (2002) Attentional modulation in visual cortex depends on task timing. Nature 419:616-620.

Grosjean M, Rosenbaum DA, Elsinger C (2001) Timing and reaction time. J Exp Psychol Gen 130:256-272.

Gross J, Kujala J, Hamalainen M, Timmermann L, Schnitzler A, Salmelin R (2001) Dynamic imaging of coherent sources: studying neural interactions in the human brain. Proc Natl Acad Sci USA 98:694-699. 
Halsband U, Passingham RE (1985) Premotor cortex and the conditions for movement in monkeys (Macaca mulatta). Behav Brain Res 18:269-276.

Hämäläinen MS, Ilmoniemi RJ (1994) Interpreting magnetic fields of the brain: minimum norm estimates. Med Biol Eng Comput 32:35-42.

Hanslmayr S, Klimesch W, Sauseng P, Gruber W, Doppelmayr M, Freunberger R, Pecherstorfer T (2005) Visual discrimination performance is related to decreased alpha amplitude but increased phase locking. Neurosci Lett 375:64-68.

Hollerman JR, Schultz W (1998) Dopamine neurons report an error in the temporal prediction of reward during learning. Nat Neurosci 1:304-309.

Jäncke L, Loose R, Lutz K, Specht K, Shah NJ (2000) Cortical activations during paced finger-tapping applying visual and auditory pacing stimuli. Brain Res Cogn Brain Res 10:51-66.

Janssen P, Shadlen MN (2005) A representation of the hazard rate of elapsed time in macaque area LIP. Nat Neurosci 8:234-241.

Jantzen KJ, Steinberg FL, Kelso JA (2005) Functional MRI reveals the existence of modality and coordination-dependent timing networks. NeuroImage 25:1031-1042.

Kaiser J, Birbaumer N, Lutzenberger W (2001) Event-related beta desynchronization indicates timing of response selection in a delayed-response paradigm in humans. Neurosci Lett 312:149-152.

Kim SG, Ashe J, Georgopoulos AP, Merkle H, Ellermann JM, Menon RS, Ogawa S, Ugurbil K (1993) Functional imaging of human motor cortex at high magnetic field. J Neurophysiol 69:297-302.

Komura Y, Tamura R, Uwano T, Nishijo H, Kaga K, Ono T (2001) Retrospective and prospective coding for predicted reward in the sensory thalamus. Nature 412:546-549.

Lange K, Rösler F, Roder B (2003) Early processing stages are modulated when auditory stimuli are presented at an attended moment in time: an event-related potential study. Psychophysiology 40:806-817.

Leon MI, Shadlen MN (2003) Representation of time by neurons in the posterior parietal cortex of the macaque. Neuron 38:317-327.

Los SA, Heslenfeld DJ (2005) Intentional and unintentional contributions to nonspecific preparation: electrophysiological evidence. J Exp Psychol Gen 134:52-72.

Macar F, Vidal F (2003) The CNV peak: an index of decision making and temporal memory. Psychophysiology 40:950-954.

Macar F, Vidal F, Casini L (1999) The supplementary motor area in motor and sensory timing: evidence from slow brain potential changes. Exp Brain Res 125:271-280.

Macar F, Lejeune H, Bonnet M, Ferrara A, Pouthas V, Vidal F, Maquet P (2002) Activation of the supplementary motor area and of attentional networks during temporal processing. Exp Brain Res 142:475-485.

MacDonald CJ, Meck WH (2004) Systems-level integration of interval timing and reaction time. Neurosci Biobehav Rev 28:747-769.

Makeig S, Westerfield M, Jung TP, Enghoff S, Townsend J, Courchesne E, Sejnowski TJ (2002) Dynamic brain sources of visual evoked responses. Science 295:690-694.

Mauk MD, Buonomano DV (2004) The neural basis of temporal processing. Annu Rev Neurosci 27:307-340.

Mauritz KH, Wise SP (1986) Premotor cortex of the rhesus monkey: neuronal activity in anticipation of predictable environmental events. Exp Brain Res 61:229-244.

Miniussi C, Wilding EL, Coull JT, Nobre AC (1999) Orienting attention in time. Modulation of brain potentials. Brain 122:1507-1518.

Müller-Gethmann H, Ulrich R, Rinkenauer G (2003) Locus of the effect of temporal preparation: evidence from the lateralized readiness potential. Psychophysiology 40:597-611.
Naccache L, Blandin E, Dehaene S (2002) Unconscious masked priming depends on temporal attention. Psychol Sci 13:416-424.

Niki H, Watanabe M (1979) Prefrontal and cingulate unit activity during timing behavior in the monkey. Brain Res 171:213-224.

Oostenveld R, Praamstra P (2001) The five percent electrode system for high resolution EEG and ERP measurements. Clin Neurophysiol 112:713-719.

Pfeuty M, Ragot R, Pouthas V (2003) When time is up: CNV time course differentiates the roles of the hemispheres in the discrimination of short tone durations. Exp Brain Res 151:372-379.

Pfeuty M, Ragot R, Pouthas V (2005) Relationship between CNV and timing of an upcoming event. Neurosci Lett 382:106-111.

Pouthas V, Garnero L, Ferrandez AM, Renault B (2000) ERPs and PET analysis of time perception: spatial and temporal brain mapping during visual discrimination tasks. Hum Brain Mapp 10:49-60.

Rao SM, Mayer AR, Harrington DL (2001) The evolution of brain activation during temporal processing. Nat Neurosci 4:317-323.

Reutimann J, Yakovlev V, Fusi S, Senn W (2004) Climbing neuronal activity as an event-based cortical representation of time. J Neurosci 24:3295-3303.

Ruchkin DS, McCalley MG, Glaser EM (1977) Event related potentials and time estimation. Psychophysiology 14:451-455.

Salmelin R, Hari R (1994) Spatiotemporal characteristics of sensorimotor neuromagnetic rhythms related to thumb movement. Neuroscience 60:537-550.

Salmelin R, Hämäläinen M, Kajola M, Hari R (1995) Functional segregation of movement-related rhythmic activity in the human brain. NeuroImage 2:237-243.

Schubotz RI, von Cramon DY (2001) Interval and ordinal properties of sequences are associated with distinct premotor areas. Cereb Cortex $11: 210-222$.

Schubotz RI, von Cramon DY (2003) Functional-anatomical concepts of human premotor cortex: evidence from fMRI and PET studies. NeuroImage 20 [Suppl 1]:S120-S131.

Schubotz RI, Friederici AD, von Cramon DY (2000) Time perception and motor timing: a common cortical and subcortical basis revealed by fMRI. NeuroImage 11:1-12.

Stancak Jr A, Pfurtscheller G (1995) Desynchronization and recovery of beta rhythms during brisk and slow self-paced finger movements in man. Neurosci Lett 196:21-24.

Suri RE, Schultz W (2001) Temporal difference model reproduces anticipatory neural activity. Neural Comput 13:841-862.

Toni I, Schluter ND, Josephs O, Friston K, Passingham RE (1999) Signal-, set-, and movement-related activity in the human brain: an event-related fMRI study. Cereb Cortex 9:35-49.

Trillenberg P, Verleger R, Wascher E, Wauschkuhn B, Wessel K (2000) $\mathrm{CNV}$ and temporal uncertainty with aging and non-aging S1-S2 intervals. Clin Neurophysiol 111:1216-1226.

van Burik M, Pfurtscheller G (1999) Functional imaging of postmovement beta event-related synchronization. J Clin Neurophysiol 16:383-390.

Walter WG, Cooper R, Aldridge VJ, McCallum WC, Winter AL (1964) Contingent negative variation: an electric sign of sensorimotor association and expectancy in the human brain. Nature 203:380-384.

Worden MS, Foxe JJ, Wang N, Simpson GV (2000) Anticipatory biasing of visuospatial attention indexed by retinotopically specific alpha-band electroencephalography increases over occipital cortex. J Neurosci 20: RC63(1-6). 